\title{
Aspectos metodológicos do levantamento epidemiológico das condições de saúde bucal e qualidade da assistência odontológica entre escolares
}

\author{
Methodological aspects of the epidemiological survey of oral health conditions and quality \\ of dental care among students
}

Aspectos metodológicos de la encuesta epidemiológica de las condiciones de salud bucal y la calidad de la atención dental entre estudiantes

Andréa Maria Eleutério de Barros Lima Martins ${ }^{1,2 *}$, Amanda Neves Magalhães ${ }^{1}$, Ana Maria Rodrigues Santos $^{2}$, Fernanda Gonçalves Alves², Giovana Rafaelly Silva Antunes², Gisely Cardoso Dourado Coutinho $^{2}$, Henrique Carvalho de Brito Fernandes ${ }^{2}$, Juliana Pereira dos Santos ${ }^{1}$, Maria Alice Aguiar Soares ${ }^{2}$, Tamires Aparecida Ramos Antunes ${ }^{1}$.

\section{RESUMO}

Objetivo: Apresentar aspectos metodológicos utilizados para realizar o "Levantamento epidemiológico sobre condições de saúde bucal entre escolares de uma cidade polo de médio porte populacional situada no Norte de Minas Gerais, Brasil" que propõe avaliar as condições da saúde bucal e a qualidade do atendimento odontológico. Métodos: Estudo metodológico referente a criação e validação de instrumentos relacionados à saúde bucal e investigação epidemiológica. Resultados: Foi estimada uma amostra probabilística complexa por conglomerados aleatória simples estratificada (5,12 e 15 anos). O planejamento incluiu a condução de entrevistas e exames bucais por profissionais calibrados e acadêmicos treinados. Foram avaliadas condições normativas de saúde bucal, condições subjetivas de saúde bucal, assim como o uso e acesso dos serviços, o nível de letramento e a qualidade da assistência odontológica. Conclusão: Com a metodologia descrita será possível obter resultados que favorecem dimensionar a necessidade de ações abrangentes viabilizando melhorias nas estratégias de inclusão dos grupos populacionais mais afetados nas prioridades municipais dos serviços de saúde.

Palavras-chave: Saúde bucal, Pesquisa sobre serviços de saúde, Letramento em saúde.

\section{ABSTRACT}

Objective: To present methodological aspects used to carry out the "Epidemiological survey on oral health conditions among schoolchildren in a medium-sized pole city located in the North of Minas Gerais, Brazil" that proposes to evaluate oral health conditions and the quality of dental care. Methods: Methodological study referring to the creation and validation of instruments related to oral health and epidemiological investigation. Results: A complex probabilistic sample was estimated by simple stratified random clusters $(5,12$ and 15 years). The planning included conducting interviews and oral exams by trained professionals and trained academics. Normative oral health conditions, subjective oral health conditions, as well as the use and access of services, the level of literacy and the quality of dental care were evaluated. Conclusion: With the described methodology it will be possible to obtain results that favor the dimension of the need for comprehensive actions enabling improvements in the strategies of inclusion of the population groups most affected in the municipal priorities of health services.

Keywords: Oral health, Health services research, Health literacy.

\footnotetext{
${ }^{1}$ Universidade Estadual de Montes Claros (Unimontes), Montes Claros - MG.

${ }^{*}$ E-mail: martins.andreamebl@gmail.com

${ }^{2}$ Faculdades Unidas do Norte de Minas (FUNORTE), Montes Claros - MG.
} 
RESUMEN

Objetivo: Presentar los aspectos metodológicos utilizados para la realización de la "Encuesta epidemiológica sobre condiciones de salud bucal en escolares de una ciudad polo de tamaño mediano ubicada en el norte de Minas Gerais, Brasil" que propone evaluar las condiciones de salud bucal y la calidad de la atención odontológica. Métodos: Estudio metodológico referente a la creación y validación de instrumentos relacionados con la salud bucal y la investigación epidemiológica. Resultados: Se estimó una muestra probabilística compleja mediante conglomerados aleatorios estratificados simples (5, 12 y 15 años). La planificación incluyó la realización de entrevistas y exámenes orales por parte de profesionales y académicos capacitados. Se evaluaron las condiciones normativas de salud bucal, las condiciones subjetivas de salud bucal, así como el uso y acceso a los servicios, el nivel de alfabetización y la calidad de la atención odontológica. Conclusión: Con la metodología descrita será posible obtener resultados que favorezcan la dimensión de la necesidad de acciones integrales que permitan mejorar las estrategias de inclusión de los grupos de población más afectados en las prioridades municipales de los servicios de salud.

Palabras clave: Salud oral, Investigación sobre servicios de salud, Literatura saludable.

\section{INTRODUÇÃO}

A epidemiologia tem como propósito, em populações específicas, a avaliação das condições de saúde e de seus determinantes, com aplicação prática no controle e prevenção das doenças e agravos à saúde (ANTUNES JLF e PERES MA, 2013). Os levantamentos epidemiológicos permitem a avaliação das condições de saúde e auxiliam os governos, autoridades e profissionais na formulação de políticas e programas na prevenção e estimativa da carga das doenças, na avaliação do impacto, eficácia, efetividade ou eficiência dos esforços para controlar doenças e promover a qualidade de vida entre as pessoas.

O modelo de assistência "orientado para a ação" visa identificar os determinantes sociais das iniquidades em saúde, sugerir propostas de ação, avaliação das condições de saúde e a identificação dos seus determinantes, dentre estes os estruturais. Esse modelo sugere que diferenças estruturais e individuais resultam em iniquidades em saúde (SOLAR O e IRWIN A, 2010). Já a avaliação das condições de saúde subsidia ações consistentes com a realidade orientando ações a serem desenvolvidas. Sendo assim, os levantamentos epidemiológicos são necessários.

A Organização Mundial de Saúde (OMS) propôs a padronização do planejamento, critérios de diagnóstico, análise e sumarização dos dados de levantamentos epidemiológicos sobre condições de saúde bucal e ressaltou a necessidade da condução desses levantamentos de forma periódica (WHO, 2013). A avaliação das condições de saúde bucal, assim como das características do uso dos serviços odontológicos e comportamentos relacionados à saúde bucal permitem a vigilância epidemiológica do estado de saúde da população e subsidia a implementação ou aprimoramento de políticas de saúde (RODRIGUES CC, et al., 2004).

No Brasil foram conduzidos quatro levantamentos epidemiológicos de base nacional sobre condições de saúde bucal em 1986, em 1996 em 2003/2004 e em 2010, que demonstraram a melhoria das condições de saúde bucal dos brasileiros (BRASIL, 1988; RONCALLI AG, 1998a; RONCALLI AG, 1998b; BRASIL, 1996; BRASIL, 2003; BRASIL, 2004).

Com base na proposta da Pesquisa sobre as Condições de Saúde Bucal da População Brasileira (SB Brasil), foi conduzido o "Levantamento Epidemiológico das Condições de Saúde Bucal da População de Montes Claros, Projeto SBMoc 2008/2009" com a participação de mais de 4000 pessoas nas faixas etárias de 18-36 meses, 15-19 anos, 35-44 anos, 65-74 anos e nas idades índice de 5 e 12 anos. Foram identificadas as condições de saúde bucal da população e houve ainda o pronto atendimento aos participantes nos casos indicados (MARTINS AMEBL, et al., 2012). Além das condições normativas de saúde bucal há necessidade de se avaliar questões subjetivas que subsidiem ações de promoção de saúde e prevenção das doenças visando minimizar as iniquidades em saúde. 
Ao se considerar a importância de promover a saúde bucal das populações, a educação em saúde é fundamental visando a autonomia, ou seja, no empoderamento das pessoas quanto ao autocuidado em saúde (NUTBEAM D, 2000). Uma abordagem libertária da saúde visa elevar os níveis de Letramento em Saúde (LS) das pessoas em busca do empoderamento das pessoas e a aplicação consciente dos conhecimentos e recursos disponíveis relacionados à saúde, promovendo a participação dessas pessoas no controle da sua saúde (GEIB LTC, 2012). Um modelo teórico conceitual sobre LS apresenta fatores proximais e distais determinantes ou determinados pelo LS, ele considera a influência dos conhecimentos prévios, das competências e da motivação no processo de acesso, compreensão, avaliação e aplicação das informações relacionadas à saúde (SORENSEN K, et al., 2012).

Os diferentes níveis do LS representam graus de conhecimentos e habilidades diversos, que elevam a autonomia e o empoderamento das pessoas promovendo a melhoria da saúde das pessoas. O conceito de saúde tem apresentado distintos significados ao longo dos anos e por fim foi vinculado à qualidade de vida das pessoas (BADASH I, et al., 2017; MOLD J, 2017). A qualidade da assistência à saúde e as políticas públicas têm impacto sobre o estado de saúde das pessoas (SOLAR O e IRWIN A, 2010). Há que se considerar então a avaliação da qualidade da assistência à saúde bucal, ou seja, a avaliação do processo, estrutura e desfechos (DONABEDIAN A, 1988). Os resultados dessas avaliações podem subsidiar políticas de saúde consistentes com a realidade tendo em vista a qualidade de vida.

O planejamento em saúde, deve ser baseado em resultados de pesquisas, obtidos por intermédio de instrumentos com apropriada qualidade, no que diz respeito às suas propriedades psicométricas. Um estudo DELPHI conduzido entre experts sobre a qualidade de instrumentos que avaliam eventos relacionados à saúde propõe a verificação de 9 propriedades de medição agrupadas em 3 domínios: confiabilidade, validade e responsividade, além da interpretabilidade dos instrumentos na avaliação dessa qualidade (MOKKINK LB, et al., 2016). Não foram encontrados instrumentos aptos a avaliar níveis do Letramento em Saúde Bucal (LSB) entre escolares, assim como para avaliar a satisfação com serviço odontológico, ou seja, a qualidade da assistência odontológica ofertada que consideraram os critérios de avaliação de qualidade (MOKKINK LB, et al., 2016).

Em estudos epidemiológicos há que se ponderar a qualidade dos instrumentos de avaliação disponíveis na literatura ou desenvolver instrumentos adequados quanto à sua qualidade e aptos a avaliar o que se propõe. Nos levantamentos epidemiológicos, a coleta e a construção do banco de dados são etapas complexas e dispendiosas que demandam tempo e energia dos pesquisadores. A coleta dos dados em papel requer a digitação desses dados, atividade que deve ser conduzida por pessoal treinado. O desenvolvimento de programas de computador para a coleta de dados e concomitante alimentação da base de dados dispensam a digitação dos dados após sua coleta utilizando instrumentos impressos em papel (PEREIRA RC e MOREIRA PRC, 2009).

Diante do exposto, decorridos aproximadamente dez anos do primeiro Levantamento Epidemiológico das Condições de Saúde Bucal da População de um município de médio porte populacional do Norte de Minas Gerais (MARTINS AMEBL, et al., 2012) tem-se como objetivo apresentar os aspectos metodológicos da condução do Levantamento epidemiológico das condições de saúde bucal e qualidade da assistência odontológica entre escolares de uma cidade polo do Norte de Minas Gerais - Brasil em 2018/2019.

\section{MÉTODOS}

O projeto SBMoc 2018/2019 consistiu em um estudo epidemiológico transversal sobre as condições de saúde bucal dos escolares da população de um município de médio porte populacional do Norte de Minas Gerais. Foi preconizado por pesquisadores da Unimontes em colaboração com a Prefeitura Municipal da cidade em questão. Objetivou avaliar as condições a qualidade da saúde bucal e do atendimento odontológico, levando-se em conta a criação e validação de instrumentos específicos para estes fins.

Foi estimada uma amostra aleatória simples probabilística por conglomerados em dois estágios, estratificada por idades índices. A coleta de dados foi realizada por cirurgiões-dentistas/auxiliares de saúde bucal e acadêmicos dos cursos de Odontologia. 
Para a otimização da coleta de dados foi criado um software Sistema de Gerenciamento de Pesquisas (SGP) abordando todos os dados a serem investigados, condições normativas de saúde bucal e subjetivas. Os resultados serão avaliados com base em pressupostos estatísticos e serão divulgados entre a comunidade acadêmica, população local e a leiga podendo gerar reflexão sobre uma maior e melhor cobertura de saúde.

Após o processo de busca ativa nas escolas sorteadas, os participantes foram devidamente esclarecidos a respeito da pesquisa. O projeto de pesquisa foi aprovado pelo Comitê de Ética em Pesquisa da Universidade Estadual de Montes Claros por meio do parecer no 2.483.638, certificado de apresentação de apreciação ética: 82447617.7.0000.5146 (BRASIL, 2013).

\section{RESULTADOS}

O cálculo amostral considerou a prevalência de ocorrência do evento de $50 \%$, um nível de confiança de $95 \%(Z=1,96)$, um erro de $5-10 \%$. Foram considerados ainda os resultados do projeto SBMOC $2008 / 2009$ do índice de dentes permanentes Cariados Perdidos e Obturados (CPOD) e dentes decíduos cariados, extraídos e obturados (CEO) médios com seus respectivos desvios padrões, um nível de confiança de $95 \%(Z=1,96)$ e um erro de $5-10 \%$, taxa de não resposta de $10 \%$, identificado nas idades índice preconizadas (OLIVEIRA LFB, 2012; PALMA ABO, et al., 2012; SILVEIRA MF, et al., 2012).

Para cada estrato ou idade índice foram calculados dois tamanhos de amostras, um considerando a proporção de $50 \%$ e outro considerando a distribuição média da cárie e optou-se por considerar a estimativa que propõe a avaliação do maior número de escolares. O tamanho amostral foi corrigido pelo efeito de desenho (deff=2) e uma Taxa de Não Resposta de 10\% (Quadro 1).

Quadro 1- Fórmulas consideradas nas estimativas das amostras para a três idades índices 5, 12 e 15 anos considerando a ocorrência do evento em $50 \%$ da população e a distribuição média da cárie, município de médio porte populacional do Norte de MG, Brasil 2018/2019.

\begin{tabular}{|c|c|}
\hline Ocorrência do evento em $50 \%$ da população & Distribuição média da cárie \\
\hline $\begin{array}{l}\text { Fórmula } \\
\end{array}$ & Fórmula \\
\hline $\mathrm{x}=\frac{(\mathrm{N})\left(\mathrm{Z}^{2}\right)(\mathrm{p})(1-\mathrm{p})}{\left(\left(\mathrm{e}^{2}\right)(\mathrm{N}-1)\right)+\left(\left(\mathrm{Z}^{2}\right)(\mathrm{p})(1-\mathrm{p})\right)}$ & $\mathrm{x}=\frac{(\mathrm{N})\left(\mathrm{Z}^{2}\right)\left(\mathrm{s}^{2}\right)}{\left(\left(\mathrm{e}^{2}\right)(\mathrm{N}-1)\right)+\left(\left(\mathrm{Z}^{2}\right)\left(\mathrm{s}^{2}\right)\right)}$ \\
\hline $\begin{array}{c}\text { Onde } \\
N=3310 \\
Z=1,96 \\
p=0,5 \\
1-p=0,5 \\
e=0,05\end{array}$ & $\begin{array}{c}\text { Onde } \\
\mathrm{N}=3310 / 5 \text { ANOS } \mathrm{N}=4458 / 12 \text { ANOS } \\
\mathrm{N}=4524 / 15 \text { ANOS } \\
\mathrm{Z}=1,96 \\
\mathrm{~s}^{2}=2,573 / 5 \text { anos } 1,668 / 12 \text { anos } \\
2,881 / 15 \text { anos } \\
\mathrm{e}=0,10\end{array}$ \\
\hline $\mathrm{n}=((x)($ deff $f))+((x)($ TNR $))$ & $\mathrm{n}=((x)($ deff $f))+((x)($ TNR $))$ \\
\hline 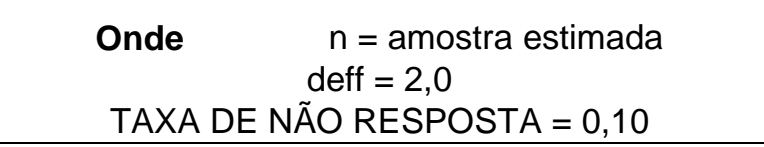 & Onde $\begin{aligned} \mathrm{n} & =\text { amostra estimada } \\
\text { deff } & =2,0 \\
\mathrm{TNR} & =0,10\end{aligned}$ \\
\hline
\end{tabular}

Fonte: Martins AMEBL, et al., 2020.

As condições de saúde bucal investigadas em sua grande maioria consideram a proposta da OMS de 2013. Foram avaliadas: condição da coroa dentária; necessidade de tratamento dentário; lesões de erosão e abrasão; traumas dentários; fluorose dentária; índice de placa visível; alterações gengivais / sangramento à sondagem; presença de cálculo dentário; bolsa periodontal (15 anos); perda de inserção periodontal (15 anos); prevalência de oclusopatias; lesões de mucosa bucal e região peribucal; necessidade de intervenções de urgência e de intervenções sistêmicas. Além das condições preconizadas pela OMS às avaliações das seguintes condições bucais foram incluídas: cálculo dentário, oclusopatias, necessidade de próteses odontológicas (WHO, 1997; WHO, 2013) (Quadro 2). 
Quadro 2 - Condições bucais consideradas no levantamento epidemiológico das condições de saúde bucal e qualidade da assistência odontológica entre escolares de município de médio porte populacional do Norte de MG, Brasil 2018/2019.

\begin{tabular}{|l|l|l|l|}
\hline \multicolumn{1}{|c|}{ Idade Índice } & $\mathbf{5}$ anos & $\mathbf{1 2}$ anos & $\mathbf{1 5}$ anos \\
\hline Condição dentária & & & \\
\hline \multicolumn{1}{|c|}{ Coroa } & & & \\
\hline & Lesão Cervical Não Cariosa & & \\
\hline Trauma Dentário & & & \\
\hline Fecessidade de tratamento & & & \\
\hline Condição Periodontal & & & \\
\hline Cálculo & Alteração Gengival & & \\
\hline Bolsa Periodontal & & & \\
\hline Perda de Inserção Periodontal PIP & & & \\
\hline Maloclusão & & & \\
\hline Necessidade de prótese & & & \\
\hline Lesões cancerizáveis & & & \\
\hline Intervenção de Urgência & & & \\
\hline
\end{tabular}

Fonte: Martins AMEBL, et al., 2020.

Após a criação e validação de instrumentos para alcançar os objetivos foi proposta uma entrevista contendo questões sobre: dados socioeconômicos, condições subjetivas de saúde, uso/avaliação dos serviços odontológicos, avaliação do letramento quanto a saúde bucal e hábitos alimentares. Além disso, propôs-se a avaliação do impacto da saúde geral e bucal na qualidade de vida, incluindo variáveis referentes a hábitos de estilo de vida (atividade física, tabagismo, estilismo, higiene bucal) ao capital social e a avaliação normativa da condição bucal.

$\mathrm{Na}$ avaliação das condições subjetivas o entrevistado classifica sua saúde bucal, a aparência dos seus dentes e gengiva bem como mastigação e relata como a saúde bucal afeta o seu relacionamento social. $O$ OHIP-14 (Oral Health Impact Profile ou Perfil do Impacto da Saúde Bucal) foi considerado conforme modificação do instrumento original traduzido para o português (OLIVEIRA BH e NADANOVSKY P, 2005).

O OHIP-14 aborda se nos últimos 6 meses, o entrevistado, apresentou problemas devido seus dentes, boca ou dentadura, que interferissem na fala, percepção do sabor dos alimentos, dor nos dentes ou boca, incômodo, dificuldade ou interrupção na alimentação, preocupação, estresse, dificuldade em relaxar, envergonhamento, irritação, dificuldade na realização das atividades diárias, sensação de piora na vida em geral, e incapacidade total de executar atividades diárias.

$\mathrm{Na}$ avaliação da autopercepção da necessidade de tratamento odontológico, acesso e uso indagou-se ao entrevistado se ele considera que necessita de atendimento, e se sim qual seria o principal motivo dentre os apresentados (revisão, prevenção, limpeza; sangramento gengival; dor de dente; dor na gengiva; colocar aparelho ortodôntico; necessidade de prótese, dentadura, coroa, ponte; dor muscular próximo ao ouvido; necessidade de canal; restaurações ou obturação; mau hálito; extração dentária; clareamento) além da alternativa outros e espaço para especificação do motivo.

A investigação das condições normativas se baseou em exames bucais. Foi proposta a avaliação dos dentes, da oclusão de lesões de mucosa e região peribucal com o registro da região anatômica e o tipo de lesão fundamental / variações da normalidade. A condição de saúde bucal e sua localização foram registradas utilizando os códigos recomendados pela OMS modificados (WHO, 2013).

Foi feito um processo de seleção de uma empresa a ser contratada para desenvolver um software para realizar a coleta e construção simultâneas do banco de dados. Foi previsto o desenvolvimento do Sistema de Gerenciamento em Pesquisa (SGP). O programa foi instalado em dispositivos portáteis, tablets. Foi, também, idealizada a transferência, via rede local sem fios (wireless), dos dados armazenados no tablet para a base de dados. O SGP será submetido ao registro de direitos autorais. 
O estudo piloto foi conduzido em uma população distinta da que está sendo avaliada. Foram desenvolvidos os instrumentos de avaliação do LS e satisfação com a assistência odontológica. Será conduzida a avaliação da qualidade dos instrumentos para estimar os níveis do LS e satisfação com a assistência odontológica considerados no estudo piloto (MOKKINK LB, et al., 2016).

O viés de aferição foi minimizado por meio do treinamento e calibração conduzidos em conformidade com os métodos recomendados. As equipes de campo foram treinadas visando assegurar um grau aceitável de uniformidade nas entrevistas e exames. A coleta de dados tem sido conduzida por 22 cirurgiões-dentistas e por acadêmicos do curso de Odontologia.

A calibração foi conduzida em três etapas: 1) Treinamento teórico com exposição teórica e dialogada dos critérios de diagnóstico por meio de projeções de apresentações em PowerPoint e de manuais. 2) Treinamento prático com exames em voluntários selecionados conforme normas de biossegurança vigentes. Para evitar cansaço e desconforto aos voluntários cada um foi examinado no máximo cinco vezes. 3) Os examinadores foram treinados e calibrados com intervalo de sete dias entre o treinamento e calibração viabilizando a assimilação dos critérios de diagnóstico considerados na pesquisa. Foram estimadas as concordâncias intraexaminador e interexaminadores.

Os assuntos foram retomados em treinamento teórico ou prático, em casos de discrepâncias, para que as diferenças fossem revisadas e resolvidas após discussão em grupo. Após padronização no uso de critérios de diagnóstico para as condições bucais investigadas mediante estimativas das concordâncias obtidas por meio dos coeficientes Kappa (variáveis nominais: condições da coroa dentária, necessidade de tratamento dentário, alteração gengival, uso e necessidade de prótese) e Kappa Ponderado (variáveis ordinais: fluorose, oclusão, cálculo dentário, bolsa e perda de inserção).

O projeto tem sido divulgado para comunidade escolar e familiares. Após o processo de busca ativa nas escolas sorteadas, os participantes foram devidamente esclarecidos a respeito da pesquisa. Foram solicitados os assentimentos entre os escolares de 12 e 15 anos e o consentimento aos maiores de idade responsáveis pelos menores. Após sensibilização dos gestores e familiares dos escolares foram realizadas as entrevistas e exames conforme agendamento com os gestores das escolas.

O exame foi realizado em ambientes amplos sob boas condições de iluminação, com a utilização de espátula de madeira, espelho bucal plano e sonda específica preconizada pela Organização Mundial da Saúde (WHO, 2013). Os exames intrabucais tem sido realizado nos pátios das instituições de ensino. Durante a coleta de dados também houve distribuição de cartilhas com informações sobre saúde bucal e entrega de escovas de dentes aos entrevistados. Os escolares cujos exames revelaram alterações na mucosa foram encaminhados para as clínicas de Estomatologia da Unimontes para diagnóstico, condutas e/ou tratamentos adequados como de urgência e tratamento sistêmico.

As análises foram conduzidas conforme pressupostos estatísticos, na descritiva foram estimadas as medidas de tendência central, variabilidade e frequência; nas bivariadas e múltiplas foram estimadas associações das variáveis investigadas com distintos desfechos. Os resultados da pesquisa serão divulgados entre os gestores das Secretarias Municipais de Saúde e Educação, o que contribuirá para o direcionamento das ações em saúde e inclusão dos grupos populacionais mais afetados nas prioridades municipais. Se necessário será proposta a condução de oficinas visando o planejamento e execução de atividades para aumentar os níveis de LS dos escolares e de seus familiares.

\section{DISCUSSÃO}

Os quatros grandes levantamentos epidemiológicos realizados na população brasileira foram importantes para conhecer as necessidades e as condições de saúde bucal no país (BRASIL, 1988; RONCALLI AG, 1998a; RONCALLI AG, 1998b; BRASIL, 1996; BRASIL, 2004; BRASIL, 2012). Ao considerar estudos transversais, como os levantamentos epidemiológicos, manter uma base metodológica uniforme é essencial nas estratégias de vigilância da saúde bucal. Historicamente, alguns indicadores foram desenvolvidos para 
analisar as condições bucais (cárie, doença periodontal, maloclusão, fluorose, etc.) a fim de avaliar as doenças e agravos bucais. A iniciativa da OMS levou ao aprimoramento gradual das ferramentas de pesquisa, resultando em pesquisas mundiais seguindo padrões semelhantes aos usados pela OMS (WHO, 1997; WHO, 2013).

Dessa forma, no levantamento de 1986 avaliou as variáveis cárie, doença periodontal, uso e necessidade de prótese e avaliação do serviço odontológico (BRASIL, 1986). Já em 2003, na avaliação utilizou o questionário da OMS de 1997 (WHO,1997). Além de analisar condição socioeconômica, autopercepção em saúde bucal e acesso aos serviços odontológicos quanto à satisfação da população (BRASIL, 2003).

Os índices utilizados no SB BRASIL 2010 avaliaram a condição socioeconômica, utilização de serviços, autopercepção de saúde bucal, cárie dentária, condição periodontal, tratamento dentário, oclusão, fluorose, edentulismo (uso e necessidade de prótese), para isso foi considerado o manual da OMS de1997 (WHO,1997, BRASIL, 2010). Nos projetos SB 2002/2003 (BRASIL, 2004) e SB Moc (MARTINS AMEBL, et al., 2012) avaliaram as mesmas condições do Levantamento de 1986 (RONCALLI AG, 1998b), além de condição da coroa, condição da raiz, necessidade de tratamento, fluorose, maloclusão, perda de inserção periodontal, questões subjetivas e uso de serviço.

A situação epidemiológica mostrou que, entre os adolescentes avaliados, a prevalência e severidade da cárie dentária foram altas, a taxa de edentulismo entre adultos e idosos foi elevada e houve desigualdades regionais nos dados epidemiológicos apresentados em 1986 (BRASIL, 1988). Declínio da cárie dentária e persistente desigualdade regional na população escolar foram o cenário epidemiológico em 1996 (BRASIL, 1988). Confirmou-se o declínio da cárie dentária na população escolar e a desigualdade nas principais regiões, edentulismo ainda preocupante e surgimento de outras necessidades, como a maloclusão, em 2003 (BRASIL, 2003).

Quanto à idade avaliada nos principais levantamentos epidemiológicos, apenas crianças participaram dos estudos de 1996. No ano de 1986, foram avaliados crianças e adultos, e, em 2003, idosos e bebês. Os grupos etários do SB Brasil 2010 foi de 5 anos e de 12 anos e pertencentes aos grupos etários de 15 a 19 anos, 35 a 44 anos e 65 a 74 anos. O SB 2002/2003, SB Moc, SB 2010 avaliaram bebês, crianças, adolescentes, adultos e idosos (BRASIL, 1988; BRASIL, 1996; BRASIL, 2003; MARTINS AMEBL, et al., 2012; BRASIL, 2010). No SB Moc 2018/2019 foi proposta a avaliação de crianças de 5 anos, adolescentes de 12 anos e de 15 anos.

Para se adequar a um padrão de uniformidade de critérios que oferecesse a menor margem de erro possível, no levantamento de 1986, supervisores de odontologia calibraram examinadores, orientando-os sobre os procedimentos a serem adotados durante a pesquisa (BRASIL, 1988). Em 1996, houve calibração dos examinadores em área nacional, regional, estadual e municipal, porém sem relato do cálculo de concordância e dos resultados (BRASIL, 1996). Para garantir uma interpretação unificada e consistente dos padrões padronizados de coleta de dados, profissionais do levantamento de 2003 foram treinados para atuar como instrutores de calibração. Esses palestrantes realizam seminários de calibração em cada estado com o objetivo de calibrar a equipe de campo para minimizar as diferenças entre os diferentes inspetores (BRASIL, 2003).

A calibração dos levantamentos de 2003 e de 2010 (BRASIL, 2003; BRASIL, 2012) fez o cálculo de concordância percentual ou Kappa, sendo que no SB Moc 2008/2009 também foi estimado o Kappa ponderado e o Coeficiente de Correlação Intraclasse (CCl), no entanto somente os resultados do SB Moc 2008/2009 (MARTINS AMEBL, et al., 2012) foram publicados.

A técnica de calibração utilizada no SBBrasil 2010 (BRASIL, 2012) foi uma técnica de consenso, que é utilizada para calcular o coeficiente de consenso entre os resultados obtidos por cada examinador e 0 consenso da equipe. Tomando como referência o modelo proposto pela OMS, e calculando o coeficiente Kappa ponderado para cada examinador, faixa etária e condição pesquisada, com valor limite mínimo aceitável de 0,65 (OMS, 1993). No levantamento SB Moc 2018/2019, o cálculo da concordância foi conduzido por meio do coeficiente de Kappa Simples e Ponderado. Os examinadores receberam treinamento e calibração e foram estimadas as concordâncias (reprodutibilidade) intraexaminador e interexaminadores. 


\section{CONCLUSÃO}

O levantamento epidemiológico sobre saúde bucal entre escolares de um município de médio porte populacional do Norte de MG, Brasil 2018/2019 apresenta metodologia criteriosa considerando os princípios epidemiológicos em pesquisa e seguiu a preconização do Ministério da Saúde Brasileiro e da OMS, condição fundamental para fidedignidade dos resultados, assim como a comparabilidade dos dados com outras investigações. Com a metodologia descrita será possível obter resultados que favorecem dimensionar a necessidade de ações abrangentes viabilizando melhorias nas estratégias de inclusão dos grupos populacionais mais afetados nas prioridades municipais dos serviços de saúde compatíveis com a realidade. Além disso, observar os resultados da investigação podem contribuir para a implantação de um serviço público-odontológico resolutivo. Além disso, o SGP poderá ser utilizado em outras pesquisas.

\section{AGRADECIMENTOS E FINANCIAMENTO}

Agradecemos o fomento da Fundação de Amparo à Pesquisa do Estado de Minas Gerais - FAPEMIG. Edital Programa de Pesquisa do Sistema Único de Saúde-PPSUS / Chamada FAPEMIG 007 de 2017 PROCESSO N.: CDS - APQ-03861-17).

\section{REFERÊNCIAS}

1. ANTUNES JLF, PERES M. Epidemiologia da saúde bucal. 2013; 738p.

2. BADASH I, et al. Redefining health: the evolution of health ideas from antiquity to the era of value-based care. Cureus, 2017; 9(2).

3. BRASIL. Conselho Nacional de Saúde. Resolução $n^{\circ} 466$, de 12 de dezembro de 2012. Aprova normas regulamentadoras de pesquisas envolvendo seres humanos. Brasília: Diário Oficial da União, 2013.

4. BRASIL. Levantamento Epidemiológico em Saúde bucal: 1a etapa - cárie dental - projeto. Brasília: Ministério da Saúde, 1996.

5. BRASIL. Levantamento Epidemiológico em Saúde Bucal: Brasil, zona urbana, 1986. Série C: Estudos e Projetos, 4. 1988. 137p.

6. BRASIL. Projeto SB Brasil 2003: Condições de Saúde Bucal da população brasileira. Brasília: Ministério da Saúde, 2003.

7. BRASIL. Resultados Principais do Projeto SB Brasil 2003: Condições de Saúde Bucal da população Brasileira $2002-$ 2003. Brasília: Ministério da Saúde, 2004.

8. BRASIL. SB Brasil 2010: Pesquisa Nacional de Saúde Bucal: resultados principais. Brasília: Ministério da Saúde, 2012.

9. DONABEDIAN A. The quality of care: how can it be assessed? JAMA, 1988; 260(12): 1743-8.

10. GEIB LTC. Determinantes sociais da saúde do idoso. Ciência \& Saúde Coletiva, 2012; 17(1): $123-133$.

11. MARTINS AMEBL, et al. Levantamento epidemiológico das condições de saúde bucal da população de Montes Claros - MG: Projeto SBMOC. Revista Unimontes Científica. 2012; 14(1):3-14.

12. MOKKINK LB, et al. The Consensus-based Standards for the selection of health Measurement Instruments (COSMIN) and how to select an outcome measurement instrument. Braz J PhysTher, 2016; 20(2): 105-113.

13. MOLD J. Goal-Directed Health Care: Redefining Health and Health Care in the Era of Value-Based Care. Cureus, 2017; 9(2): e1043.

14. NUTBEAM D. Health literacy as a public health goal: a challenge for contemporary health education and communication strategies into the 21st century. Health Promotion International, 2000; 15(3): 259-267.

15. OLIVEIRA LFB, et al. Levantamento das condições de Saúde Bucal em crianças de 12 anos no município de Montes Claros - MG. Revista Unimontes Científica, 2012; 14(1): 83-98.

16. OLIVEIRA BH, NADANOVSKY P. Psychometric properties of the Brazilian version of the oral Health Impact ProfileShort form. Community Dent Oral Epidemiol 2005; 33: 307-14.

17. PALMA $A B O$, et al. Saúde bucal de crianças de 5 anos de idade de Montes Claros, Brasil. Revista Unimontes Científica, 2012; 14(1):69-82.

18. PEREIRA RC, MOREIRA PRC. Sistema Automatizado para Levantamento Epidemiológico em Saúde Bucal. Universidade Estadual Paulista, 2009.

19. RODRIGUES CC, et al. Conhecimento e atitudes em saúde bucal de usuários do serviço público. Revista APS, 2004; $7(1): 8-16$.

20. RONCALLI AG. Levantamento Epidemiológico de Saúde Bucal, Brasil, 1996: Relatório Paralelo. Natal, 1998 a.

21. RONCALLI AG. Perfil Epidemiológico de Saúde Bucal no Brasil 1986-1996. Natal, 1998b.

22. SILVEIRA MF, NASCIMENTO JE, HAIKAL DS et al. Saúde bucal dos adolescentes de Montes Claros, Minas Gerais, Brasil. Revista Unimontes Científica, 2012; 14(1): 99-110.

23. SOLAR O, IRWIN A. A conceptual framework for action on the social determinants of health. Social Determinants of Health Discussion, Paper 2 (Policy and Practice). Geneva: World Health Organization, 2010.

24. SORENSEN K, et al. Health literacy and public health: a systematic review and integration of definitions and models. BMC Public Health, 2012; 12:80.

25. WORLD HEALTH ORGANIZATION (WHO). Oral health surveys: basic methods. 4th ed. Geneva: World Health Organization, 1997.

26. WORLD HEALTH ORGANIZATION (WHO). Oral health surveys: basic methods. World Health Organization, 2nd ed. Geneva: World Health Organization, 2013; $127 \mathrm{p}$. 